\title{
Multifocal Pattern Dystrophy Simulating Fundus Flavimaculatus: Multimodal Imaging for Early Diagnosis
}

\author{
Tryfon Rotsos $^{a} \quad$ Alexandra Gkounta ${ }^{b}$ Chrysanthos Symeonidis ${ }^{c}$ \\ Anastasios Lavaris $^{b}$ Emmanouil Mavrikakis ${ }^{b}$ \\ a1st Department of Ophthalmology, University of Athens, Athens, Greece; ${ }^{b}$ Department \\ of Ophthalmology, General Hospital of Athens, Athens, Greece; '2nd Department of \\ Ophthalmology, Aristotle University of Thessaloniki, Thessaloniki, Greece
}

\section{Keywords}

Multifocal pattern dystrophy simulating fundus flavimaculatus - Optical coherence tomography · Autofluoresence · Fluorescein angiography · Pattern electroretinogram

\begin{abstract}
Multifocal pattern dystrophy simulating fundus flavimaculatus (MPDSFF) is a clinical entity characterized by several clinicopathological, angiographic, tomographic, and electrophysiological findings. A 58-year-old caucasian female patient presented with bilateral floaters and metamorphopsia. Best-corrected visual acuity (VA) was $6 / 6$ in both eyes and intraocular pressure was 14 and $15 \mathrm{~mm} \mathrm{Hg}$, respectively. Fundus examination, optical coherence tomography $(\mathrm{OCT})$, autofluoresence $(\mathrm{AF})$, fluorescein angiography (FA) and pattern Electroretinogram were employed for the diagnosis of this case. Clinical and imaging findings were consistent with MPDSFF. Noticeable progression was observed in OCT scans 6 months following the baseline visit, while no significant changes were observed over the following 12 months. Prognosis of VA in MPDSFF patients may remain relatively good even in the presence of considerable anatomic changes. Disease progression may be slow and significant reduction in VA may present only secondary to a choroidal neovascular membrane. Patient follow-up should include OCT scans, PERG, and AF in addition to VA and dilated fundus examination every 6-12 months. As relevant literature is limited and no effective treatment modality has been employed for this clinical entity, the identification of the cellular death pathway in pattern dystrophies may lead to an applicable management approach.
\end{abstract}

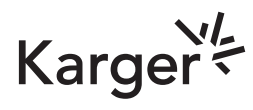



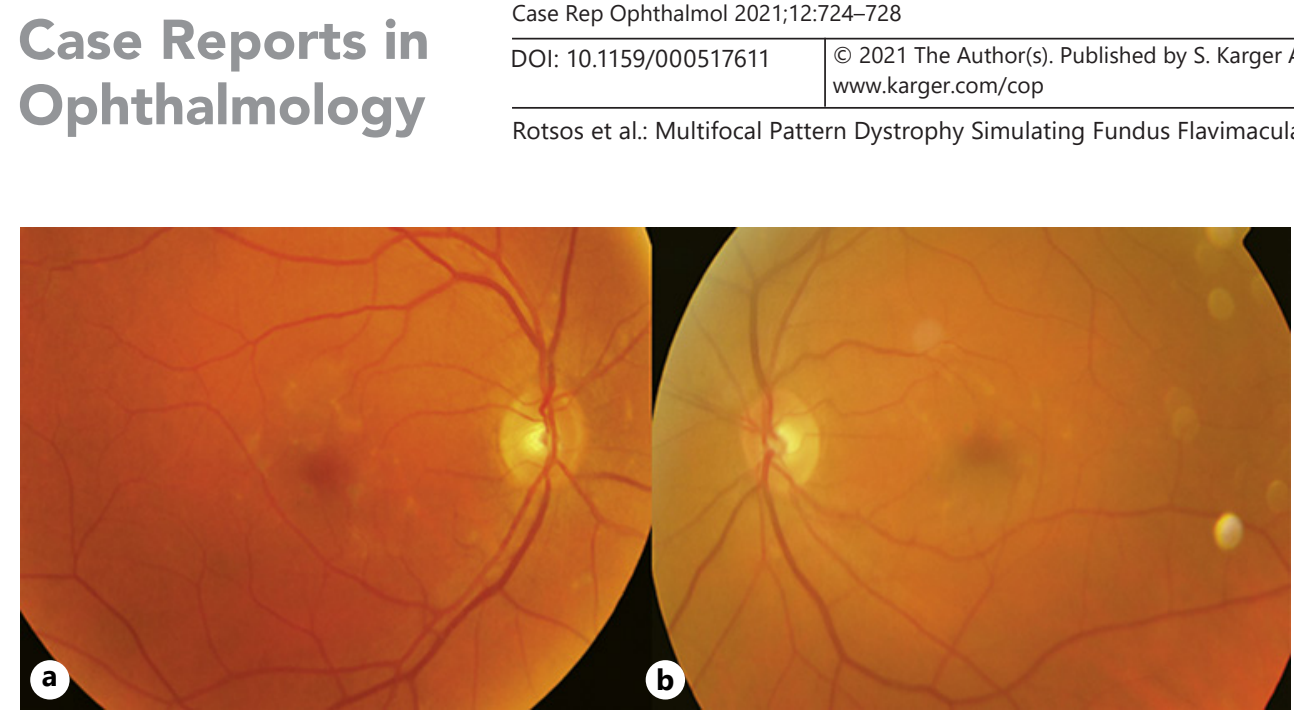

Fig. 1. a, b Fundus examination revealed white-yellowish flecks located in the posterior pole in an almost symmetrical pattern bilaterally in addition to a small number of hyperpigmented lesions.

\section{Introduction}

Multifocal pattern dystrophy simulating fundus flavimaculatus (MPDSFF) is a clinical entity characterized by several clinicopathological, angiographic, tomographic, and electrophysiological findings. Given the fact that contemporary literature on MPDSFF is very limited and focused on cases characterized by significant progression and considerable anatomic changes, the present report may contribute to early diagnosis [1].

\section{Case Presentation}

A 58-year-old Caucasian female patient presented with floaters and metamorphopsia in both eyes. Best-corrected visual acuity (VA) was $6 / 6$ bilaterally, and intraocular pressure was 14 and $15 \mathrm{~mm} \mathrm{Hg}$, respectively. Fundus examination (Fig. 1) revealed white-yellowish flecks located in the posterior pole in an almost symmetrical pattern bilaterally in addition to a small number of hyperpigmented lesions. Optical coherence tomography (OCT, Fig. 2) showed focal thickening characterized by high reflectance with disruption of the outer limiting membrane, ellipsoid zone, outer photoreceptor segments and interdigitation zone. The patient did not suffer from any systemic metabolic diseases, and no significant co-morbidity was present. Progression was observed bilaterally 6 months later. Autofluorescence (Fig. 3) revealed lesions characterized by increased autofluorescence with small adjacent areas of decreased autofluorescence within the area of the lesion. Fluorescein angiography (Fig. 4) depicted a number of hyperfluorescent lesions bilaterally, while there were no signs of "dark choroid" in the mid-periphery, a finding seen characteristically in Stargardt disease. Dark choroid describes the absence of the normal background fluorescence. The cause of this is unknown but may relate to the deposition of an abnormal material in the retinal pigment epithelial cells. This finding does not correlate with the severity or duration of disease and it is not present in all patients with heredomacular degeneration [2]. Lastly, pattern ERG was slightly decreased in both eyes (OD $155 \mu \mathrm{V} / 40.5 \mathrm{msec}$ and OS $145.5 \mu \mathrm{V} / 38.9 \mathrm{msec}$ ).

The patient's husband, daughter, and son were also examined. Husband and son had no clinical findings. Fundoscopy of the 32-year-old daughter revealed very early flecks located in the posterior pole but OCT and ERG examination were unremarkable. We keep the family members under observation and no change was noticed the following 12 months.

Clinical and imaging findings were consistent with MPDSFF. Noticeable progression was observed in OCT scans 6 months following the baseline visit while no significant changes

\section{Karger'}




\section{Case Reports in Ophthalmology}

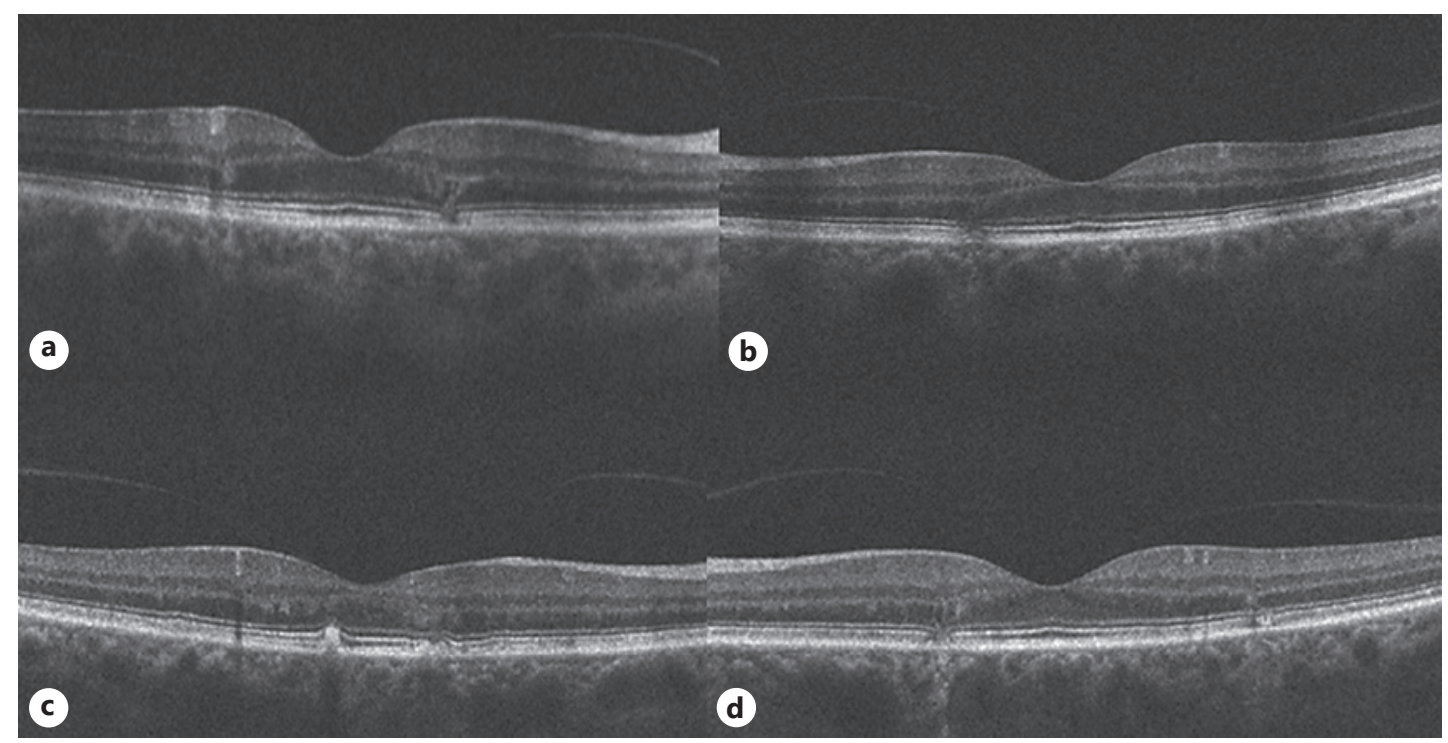

Fig. 2. OCT in the right (a) and left eye (b): focal thickening characterized by high reflectance with disruption of the outer limiting membrane, ellipsoid zone, outer photoreceptor segments, and interdigitation zone. c, d Progression was observed bilaterally 6 months later. OCT, optical coherence tomography.

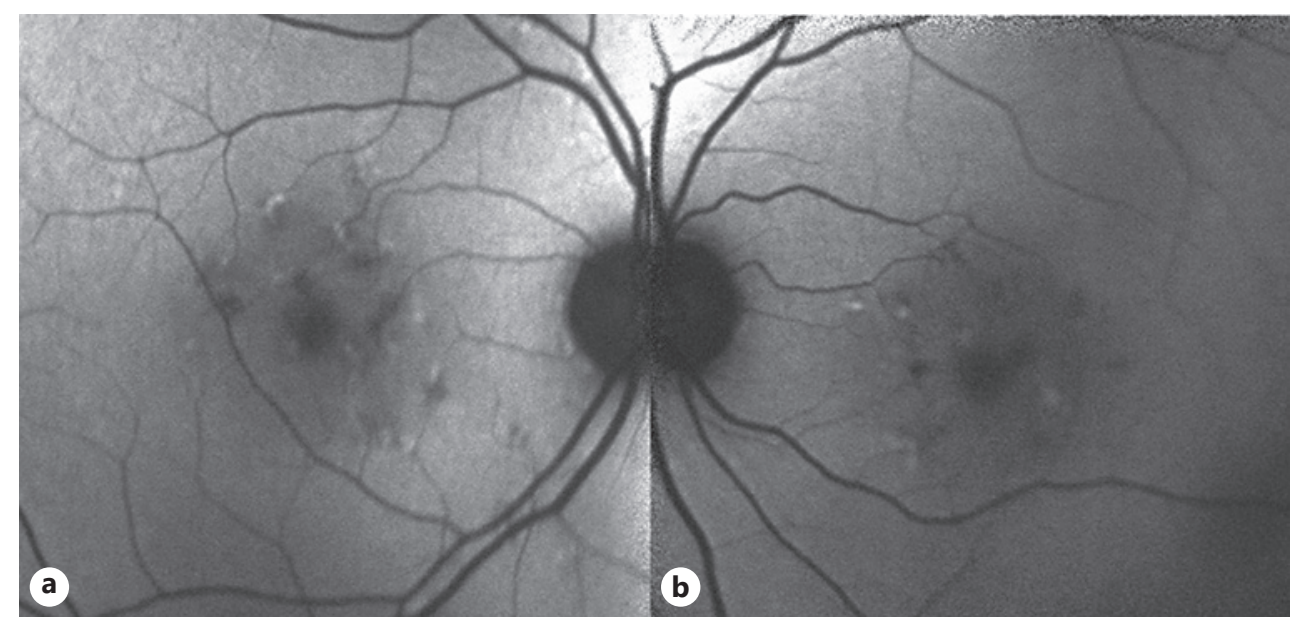

Fig. 3. AF in the right (a) and left eye (b). Lesions show increased autofluorecence with small adjacent areas with decreased autofluorecence within the area of the lesion. AF, autofluoresence.

were observed over the following 12 months. Our diagnosis was determined by multimodal imaging and ERG since no genetic testing is employed in our clinical practice.

\section{Discussion}

MPDSFF is characterized by extracellular material deposition at the level of the retinal pigment epithelium. This clinical entity is inherited due to an autosomal dominant allele, and previous studies have identified mutations in the peripherin/RDS gene as a major cause of MPDSFF [3].

In the context of MPDSFF, multiple white-yellowish central and paracentral lesions are observed. These may be scattered or partially connected (as in Stargardt's disease), but without 

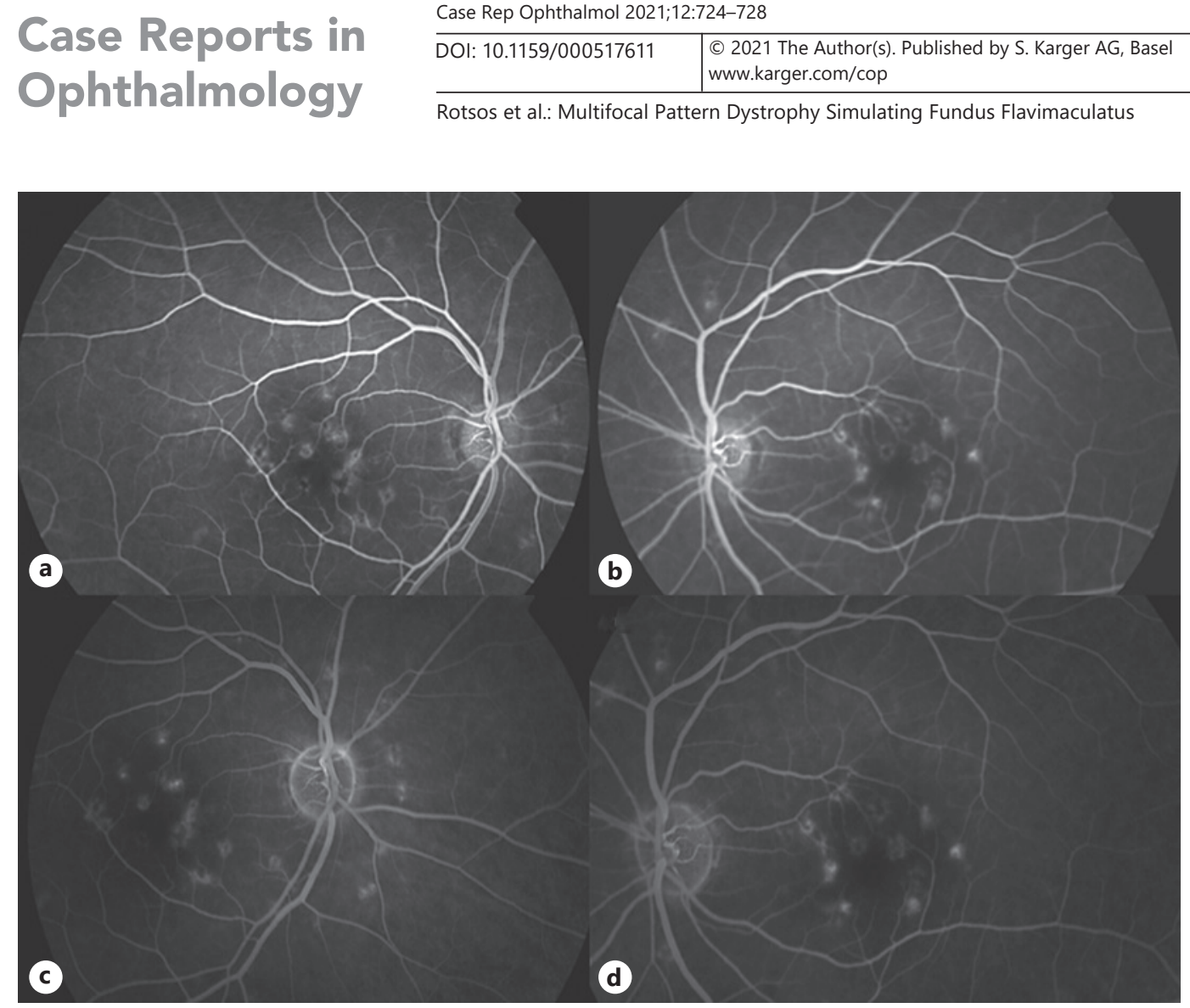

Fig. 4. Fluorescein Angiography (FA) depicted a number of hyperfluorescent lesions while there were no signs of "dark choroid" in the mid-periphery (right eye, early stages (a), left eye, early stages (b), right eye, late stages, left eye, late stages (c)).

angiographic findings of "dark choroid" in the mid-periphery from lipofuscin deposition. OCT is considered the most appropriate diagnostic modality as multiple hyper-reflective areas between the retinal pigment epithelium/Bruch's complex as well as the ellipsoid zone are revealed as was the case here. Moreover, localized discontinuities of the photoreceptor layer with the external limiting membrane are also observed. As far as angiographic findings are concerned, there are multiple hypofluorescent areas in the early phase while there is staining noted in the late phase. Pattern ERG has been shown to be more sensitive than ERG and abnormal findings may be detected even during the early stages of the disease.

Regarding prognosis, VA in MPDSFF patients may remain relatively good even in the presence of considerable anatomic changes. Disease progression may be slow and significant reduction in VA may present only secondary to a choroidal neovascular membrane. Patient follow-up should include OCT scans, PERG, and autofluoresence in addition to VA and dilated fundus examination every 6-12 months.

In the case presented here, more subtle anatomic changes were observed compared to previously reported similar cases. As relevant literature is limited and no effective treatment modality has been employed for this clinical entity, the potential identification of a specific cellular death pathway in pattern dystrophies may lead to a valid therapeutic approach.

\section{Statement of Ethics}

Written informed consent was obtained from the patient for publication of this case report and any accompanying images. Ethics approval was not required in accordance with national guidelines. 


\section{Conflict of Interest Statement}

The authors report no proprietary interest or financial support.

\section{Funding Sources}

No funding was attained for the completion of this study.

\section{Author Contributions}

Tryfon Rotsos, Alexandra Gkounta, and Anastasios Lavaris have contributed in data acquisition, Tryfon Rotsos has contributed in drafting the first version of the manuscript, Chrysanthos Symeonidis has contributed in drafting the final version of this manuscript, and Emmanouil Mavrikakis has contributed in data acquisition and in drafting the first version of the manuscript. All the authors approve the final version of this manuscript and attest that they meet the current ICMJE criteria for Authorship.

\section{Data Availability Statement}

Research data are not publicly available. Relevant enquiries can be directed to the corresponding author.

\section{References}

1 Roy R, Kumar S, Chandrasekharan DP, Ghose A, Sharma P. Multimodal imaging in multifocal pattern dystrophy simulating fundus flavimaculatus. Indian J Ophthalmol. 2016;64:395-6.

2 Fish G, Grey R, Sehmi KS, Bird AC. The dark choroid in posterior retinal dystrophies. Br J Ophthalmol. 1981; 65:359-63.

3 Boon CJ, van Schooneveld MJ, den Hollander AI, van Lith-Verhoeven JJ, Zonneveld-Vrieling MN, Theelen T, et al. Mutations in the peripherin/RDS gene are an important cause of multifocal pattern dystrophy simulating STGD1/fundus flavimaculatus. Br J Ophthalmol. 2007;91:1504-11. 